\title{
MULTI-POPULATION EVOLUTIONARY ALGORITHM FOR SOLVING CONSTRAINED OPTIMIZATION PROBLEMS
}

\author{
Ziyi Chen and Lishan Kang \\ State Key Laboratory of Software Engineering, Wuhan University, Wuhan 430072, Hubei, \\ China
}

Abstract: A novel multi-population evolutionary algorithm (MPEA) is presented, which can solve the constrained function optimization problems rather efficiently. The MPEA adopts three populations with different multi-parent crossover operators. So each population emphasizes particularly on different searching regions and the complementarity of these three crossover operators can enhances the diversity of individuals, which improves the search ability of the MPEA dramatically. And during the MPEA runs, the three populations exchange the best solution in each generation to adjust its search direction to the possible optimum solution. Experiments have been carried on several benchmark functions to test the performance of the presented MPEA. Numerical results show that MPEA is highly competitive with other algorithms in effectiveness and generality.

Key words: evolutionary algorithm,constrained function optimization,multiple populations, Multi-parent crossover operator

\section{INTRODUCTION}

Researchers have developed many evolutionary algorithms to solve the constrained optimization problems.For example, DE[1],HDE[2],MIHDE[3], GENOCOP[4],etc. Meanwhile, many strategies have been adopted to improve the performance of evolutionary algorithms $[5-8]$ 。

These algorithms are basically single population evolutionary algorithms. Such single population evolutionary algorithms are powerful and perform 
well on a wide variety of problems. However, better results can be obtained by introducing multiple subpopulations. Every subpopulation evolves over a few generations isolated before one or more individuals are exchanged between the subpopulation[9][10] .The multi-population evolutionary algorithm models the evolution of a species in a way more similar to nature than the single population evolutionary algorithm. The key task of solving constrained function optimization problems is to design universal evolutionary operator with high efficiency and scheme out suitable strategy to handle the constraints .

A novel multi-population evolutionary algorithm(MPEA), which handles the constrained function optimization problems, is presented in this paper. Three populations are created at the beginning of the MPEA run and each population uses different multi-parent crossover operators. The complementarity among three crossover operators enhances the diversity, which makes the MPEA more likely to find the global optimum than other single population evolutionary algorithms. And during the MPEA runs, different populations exchange their best solutions in each generation in order to enhance its search performance. The new approach is compared against other evolutionary optimization techniques in several benchmark functions. The results obtained show that the new approach is a general, effective and robust method. Its performance outperforms some other techniques.

\section{DESCRIPTION OF ALGORITHM (MPEA)}

\subsection{Constrained function optimization}

Without loss of generality, we assume that the objective function $f(x)$ is minimized. The maximization problem can converted into a minimization problem according to the duality principle. The nonlinear programming problem is generally described as follows:

Minimize $\quad f(x), x=\left(x_{1}, x_{2}, \ldots, x_{n}\right) \in R^{n}$

Subject to $\quad h_{i}(x)=0, i=1,2, \ldots, n_{1}$

$$
\mathrm{g}_{j}(\mathrm{x}) \leq 0, j=1,2, \ldots, n_{2}
$$

where $X^{l} \leq x=\left(x_{1}, x_{2}, \ldots, x_{v}\right) \leq X^{u}$ 
The domain space of problem: $D=\left\{x \mid X^{l} \leq x \leq X^{u}\right\}$ 。

For most difficult real world problems, the objective function, constraints $g_{j}(x)$ and $h_{k}(x)$ are nonlinear.

\subsection{The flow of MPEA}

\section{Algorithm MPEA}

\section{BEGIN}

Initialize three different populations randomly : $p_{1}, p_{2}, p_{3}$ : $p_{j}=\left\{X_{j 1}, X_{j 2}, \ldots, X_{j N}\right\}, \quad X_{j i} \in \mathrm{D} ; 1 \leq j \leq 3,1 \leq i \leq N$ generation $=0$

$X_{j, b e s t}=\arg \underset{1 \leq i \leq N}{\operatorname{Min}} f\left(X_{j i}\right) ; 1 \leq j \leq 3$

$X_{j, \text { worst }}=\arg \underset{1 \leq i \leq N}{\operatorname{Max}} f\left(X_{j i}\right) ; 1 \leq j \leq 3$

WHILE not $\left(\left(\operatorname{abs}\left(f\left(X_{1, \text { best }}\right)-f\left(X_{1, \text { worst }}\right)\right) \leq \varepsilon\right)\right.$ and

$$
\begin{gathered}
\left(a b s\left(f\left(X_{2, \text { best }}\right)-f\left(X_{2, \text { worst }}\right)\right) \leq \varepsilon\right) \text { and } \\
\left.\left(a b s\left(f\left(X_{3, \text { best }}\right)-f\left(X_{3, \text { worst }}\right)\right) \leq \varepsilon\right)\right) \text { do }
\end{gathered}
$$

BEGIN

FOR $k=1$ to 3 do BEGIN

Select $M$ individuals randomly from population $p_{k}$ and create a new individual $X$ by Multi-Parent Crossover Operator $\left(C_{k}\right)$

FOR $j=1$ to 3 do BEGIN

$\operatorname{IF} \operatorname{better}\left(X, X_{j, \text { worst }}\right)$ THEN $X_{j, \text { worst }}=X$; 
END FOR;

$$
\begin{aligned}
& X_{k, \text { best }}=\arg \underset{1 \leq i \leq N}{\operatorname{Min}} f\left(X_{k, i}\right) ; \\
& X_{k, \text { worst }}=\arg \underset{1 \leq i \leq N}{\operatorname{Max}} f\left(X_{k, i}\right) ;
\end{aligned}
$$

\section{END FOR;}

generation $=$ generation +1 ;

END;

\section{END WHILE;}

Output results;

END;

Where $N$ is population size, $\varepsilon$ is the precision of results, $X_{\text {, hest }}$ is

the individual with lowest fitness value in population $p_{j}, X_{j, \text { worst }}$ is the individual with highest fitness value in population $p_{j} . M$ is fixed as $4 . C_{1}$ is Reflect Operator, $C_{2}$ is Compact Operator, and $C_{3}$ is Expand Operator.

\subsection{Algorithm Analysis}

MPEA uses three different populations and every population use different Multi-parent crossover operators . Furthermore, every population adopts the steady elimination strategy to generate only one new individual in each generation. When one population generates a new individual, other populations will compare the new individual with the worst individual in their own population. If the new one is better than the worst, supersedes the latter. We use real-valued encoding without a mutation operator. The main idea of MPEA is that it searches the domain space to find the feasible region at first, then approaches the possible global optimum in the feasible region which have been found.

\subsubsection{Handling the constraints - Two-time Evolution}

Commonly, the equality constraints are so strong that the estimate of the feasible region is very hard, which leads to the impossibility to search the domain space at first to find the feasible region and approach the global optima in the following. The method of Two-time evolutionary is to 
transform those equality constraints to inequality constraints, then find a appropriate penalty parameter using evolutionary computation .

The flow of Two-time Evolutionary:

Step 1: transform equality constraints

$$
h_{i}(x)=0, i=1,2, \ldots, n_{1} \text { to }:-\delta \leq h_{i}(x) \leq \delta i=1,2, \ldots \ldots n_{1},
$$

where $\delta$ is a positive parameter. Generally, we set $\delta=0.01$ 。 Thus, there are $2 n_{1}+n_{2}$ inequality constraints:

$$
g_{j}^{*}(x) \leq 0, j=1,2, \ldots 2 n_{1}+n_{2}
$$

We can define:

$$
\begin{aligned}
& w_{j}(x)=\left\{\begin{array}{ll}
0 & \text { if } g_{j}^{*}(x) \leq 0 \\
g_{j}^{*}(x) & \text { otherwise }
\end{array}, \quad j=1,2, \ldots 2 n_{1}+n_{2}\right. \\
& W(x)=\sum_{j=1}^{2 n_{1}+n_{2}} w_{j}(x)
\end{aligned}
$$

Therefore, a dualistic relation better can be defined as follow:

$$
\text { better }\left(x^{\prime}, x^{\prime \prime}\right)= \begin{cases}\text { true } & \text { if } W\left(x^{\prime}\right)<W\left(x^{\prime \prime}\right) \\ \text { false } & \text { if } W\left(x^{\prime}\right)>W\left(x^{\prime \prime}\right) \\ \text { true } & \text { if }\left(W\left(x^{\prime}\right)=W\left(x^{\prime \prime}\right)\right) \wedge\left(f\left(x^{\prime}\right) \leq f\left(x^{\prime \prime}\right.\right. \\ \text { false } & \text { if }\left(W\left(x^{\prime}\right)=W\left(x^{\prime \prime}\right)\right) \wedge\left(f\left(x^{\prime}\right)>f\left(x^{\prime \prime}\right.\right.\end{cases}
$$

It means that the individual $x^{\prime}$ is better than $x^{\prime \prime}$ when better $\left(x^{\prime}, x^{\prime \prime}\right)$ is true. The feasible solutions will be better than the infeasible solutions when such method of handling the constraints adopted.

Step 2: the objective function $f(x)$ will be act as fitness function to start evolutionary using the algorithm MPEA, the evolutionary will be stopped when

$$
\left(a b s\left(f\left(X_{\text {best }}\right)-f\left(X_{\text {worst }}\right)\right) \leq \varepsilon\right)
$$


Where $\varepsilon$ is also a small positive parameter. Since we only need the orders of magnitude of the global optima, we set $\varepsilon=0.01$. After the algorithm stops, we can gain a relative good function value $f^{*}$ 。 Step 3:

$$
F(x)=f(x)+r \sum_{i=1}^{n_{1}} a b s\left(h_{i}(x)\right)
$$

will be act as the objective function to start evolutionary again, where $r=k \times f^{*}$ 。 The best result found at this time is the global optimum of the original problem. We have obtained rather good results in all experiments with setting $k=10$. The reason lies that the value of objective function is larger than the value of penalty in the neighboring region of the optimum, but the value of the objective function has little distinction from penalty in the orders of magnitude.

\subsubsection{Multi-Parent Crossover Operator}

Multi-parent crossover operators, which mean that more than two parents are involved when generating offspring, are a more flexible version, generalizing the traditional two-parent crossover of nature. Much attention has been paying on them in the field of evolutionary computation. There are various Multi-parent crossover operators with good performance for the Real-Coded evolutionary Algorithms[11][12]. Because of the complexity of the feasible region, it is impractical that a single Multi-parent crossover operator can solve various problems. So we design three different Multiparent crossover operators which have complementary features: Reflect Operator , Compact Operator,. Expand Operator .These Multi-Parent crossover operators generate offspring by linear non-convex combination of $M$ parental vectors. Figure 1 shows how to generate a new individual using these three Multi-Parent crossover operators. 


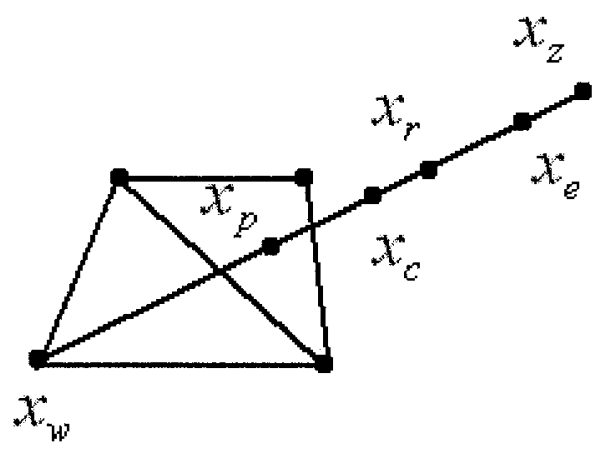

Figure. 1. Multi-Parent Crossover Operators

Their flows are as follows:

1) $M$ individuals $x_{j}, j=1,2, \ldots, M$ are randomly selected in the population.

2) find the worst individual $x_{w}$ in these $M$ individuals ( by comparing with the value of their fitness ).

3) we call the centroid of the remanent $M-1$ individuals as $x_{p}$

$$
x_{p}=\frac{\sum_{j=1}^{M} x_{j}-x_{w}}{M-1}
$$

4) find the reflecting point $x_{r}$ of $x_{w}$ corresponding to $x_{p}$ : 


$$
x_{r}=2 \times x_{w}-x_{p}
$$

We call this Crossover Operator as Reflect Operator (generate a new individual $x_{r}$ ).

5) find the reflecting point $x_{z}$ of $x_{p}$ corresponding to $x_{r}$ :

$$
x_{z}=2 \times x_{p}-x_{r}
$$

6) Select a point $x_{c}$ from $x_{p}$ to $x_{r}$ randomly, We call this Crossover Operator as Compact Operator (generate a new individual $x_{c}$ ).

7) Select a point $x_{e}$ from $x_{z}$ to $x_{r}$ randomly, We call this Crossover Operator as Expand Operator (generate a new individual $x_{e}$ ).

8 ) In these multi-parent crossover operators, $M$ is fixed to 4 . Many numerical tests have been made to find a good value of $M$. The results show that it is a good choice with setting $M=4$.

These three Multi-Parent Crossover Operators have the following important features:

- New individual generated will near the region where individuals have high fitness value, so there is rather larger probability that the new individual has higher fitness value than that of the worst individual in the population. Thus, MPEA can speedup the constringency because of the strong direction of these multi-parent crossover operators. In fact, these operators can instruct their search direction using statistical information. 
- These three crossover operators have complementary features .On the one hand, the numerical tests show that Reflect Operator is the main operator in our algorithm which can keep a good balance between exploration and exploitation. On the other hand, Compact Operator will stress on exploitation which can improve the speed of constringency . Meanwhile, Expand Operator will stress on exploration, then MPEA has lower possibility to be trapped in local optima .

- It is difficult to estimate which operators will more suitable to solve some type problems when we lack of the background of problems, so we hope that MPEA will gain higher generality because of the combination of multiple different operators .

\section{EXPERIMENTS AND RESULT}

We have developed software based on the algorithm MPEA and have made many numeric experiments .Six test problems are selected .Problem 2 to 6 are found in [14] and problem 1 to 6 in [13]. In these problems, the constraints include linear constraints, nonlinear constraints, inequality constraints and equality constraints .

In [14], Michalewicz compares the performance of six different methods on the five problems 2 to 6 . The result here is compared to the result for the best method found in [14]. In [13], Deb proposes a novel method to handle constraints, and he tested his method on nine different problems of which test problem 1 to 6 are a subset. The results obtained by the proposed method in this paper are compared to the results obtained by Deb on all these six test problems. For each of the problems, 20 independent runs are performed and the best, worst and average results are recorded.

\subsection{Benchmark problems}

1 Minimize :

$$
G_{1}(x)=5.3578547 x_{3}^{2}+0.8356891 x_{2} x_{5}+37.293239 x_{1}-40792.1
$$

Subject

to:

$$
0 \leq 85.334407+0.0056858 x_{2} x_{5}+0.00026 x_{1} x_{4}-0.0022053 x_{3} x_{5} \leq 92
$$


$90 \leq 80.51246+0.0071317 x_{2} x_{5}+0.0029955 x_{1} x_{2}+0.0021813 x_{3}^{2} \leq 110$

$20 \leq 9.300961+0.0047026 x_{3} x_{5}+0.0012547 x_{1} x_{3}+0.0019085 x_{3} x_{4} \leq 25$

where $78 \leq x_{1} \leq 102,33 \leq x_{2} \leq 45,27 \leq x_{i} \leq 45, i=3,4,5$

2 Minimize

$$
G_{2}(x)=5 x_{1}+5 x_{2}+5 x_{3}+5 x_{4}-5 \sum_{i=1}^{4} x_{i}^{2}-\sum_{i=5}^{13} x_{i}
$$

subject to $2 x_{1}+2 x_{2}+x_{10}+x_{11} \leq 10,2 x_{1}+2 x_{3}+x_{10}+x_{11} \leq 10$, $2 x_{2}+2 x_{3}+x_{11}+x_{12} \leq 10, \quad-8 x_{1}+x_{10} \leq 0,-8 x_{2}+x_{11} \leq 0$, $-8 x_{3}+x_{12} \leq 0, \quad-2 x_{4}-x_{5}+x_{10} \leq 0,-2 x_{6}-x_{7}+x_{11} \leq 0$, $-2 x_{8}-x_{9}+x_{12} \leq 0$,

Where the bounds are $0 \leq x_{i} \leq 1 \quad, \quad i=1,2, \ldots, 9$, $0 \leq x_{i} \leq 100, i=10,11,12$ and $0 \leq x_{13} \leq 1$ 。

3 Minimize

$$
G_{3}(x)=x_{1}+x_{2}+x_{3}
$$

subject to: $1-0.0025\left(x_{4}+x_{6}\right) \geq 0,1-0.0025\left(x_{5}+x_{7}-x_{4}\right) \geq 0$, $1-0.01\left(x_{8}-x_{5}\right) \geq 0$

$x_{1} x_{6}-833.33252 x_{4}-100 x_{1}+83333.333 \geq 0$

$x_{2} x_{7}-1250 x_{5}-x_{2} x_{4}+1250 x_{4} \geq 0$

$x_{3} x_{8}-1250000-x_{3} x_{5}+2500 x_{5} \geq 0$

where

$100 \leq x_{1} \leq 10000,1000 \leq x_{i} \leq 10000, i=2,3,10 \leq x_{i} \leq 1000, i=4,5, \ldots, 8$ 
4 Minimize

$$
\begin{aligned}
& G_{4}(x)=\left(x_{1}-10\right)^{2}+5\left(x_{2}-12\right)^{2}+x_{3}^{4}+3\left(x_{4}-11\right)^{2}+10 x_{5}^{6}+7 x_{6}^{2}-1 \\
& -4 x_{6} x_{7}-10 x_{6}-8 x_{7}
\end{aligned}
$$

subject

$127-2 x_{1}^{2}-3 x_{2}^{4}-x_{3}-4 x_{4}^{2}-5 x_{5} \geq 0$

$282-7 x_{1}-3 x_{2}-10 x_{3}^{2}-x_{4}+x_{5} \geq 0 \quad, 196-23 x_{1}-x_{2}^{2}-6 x_{6}^{2}+8 x_{7} \geq 0$, $-4 x_{1}^{2}-x_{2}^{2}+3 x_{1} x_{2}-2 x_{3}^{2}-5 x_{6}+11 x_{7} \geq 0$

where $-10 \leq x_{i} \leq 10, i=1,2, \ldots, 7$

5 Minimize:

$$
G_{5}(x)=e^{x_{1} x_{2} x_{3} x_{4} x_{5}}
$$

subject to: $\sum_{i=1}^{5} x_{i}^{2}=10, \quad x_{2} x_{3}-5 x_{4} x_{5}=0, x_{1}^{3}+x_{2}^{3}=-1$

where $-2.3 \leq x_{i} \leq 2.3, i=1,2,-3.2 \leq x_{i} \leq 3.2, i=3,4,5$

6 Minimize:

$$
\begin{aligned}
& G_{6}(x)=x_{1}^{2}+x_{2}^{2}+x_{1} x_{2}-14 x_{1}-16 x_{2}+4\left(x_{4}-5\right)^{2}+\left(x_{5}-3\right)^{2} \\
& +2\left(x_{6}-1\right)^{2}+5 x_{7}^{2}+7\left(x_{8}-11\right)^{2}+2\left(x_{9}-10\right)^{2}+\left(x_{10}-7\right)^{2}+45
\end{aligned}
$$

Subject to

$$
105-4 x_{1}-5 x_{2}+3 x_{7}-9 x_{8} \geq 0,-10 x_{1}+8 x_{2}+17 x_{7}-2 x_{8} \geq 0
$$




$$
\begin{aligned}
& 8 x_{1}-2 x_{2}-5 x_{9}+2 x_{10}+12 \geq 0 \\
& -3\left(x_{1}-2\right)^{2}-4\left(x_{2}-3\right)^{2}-2 x_{3}^{2}+7 x_{4}+120 \geq 0 \text {, } \\
& -5 x_{1}^{2}-8 x_{2}-\left(x_{3}-6\right)^{2}+2 x_{4}+40 \geq 0 \\
& -x_{1}^{2}-2\left(x_{2}-2\right)^{2}+2 x_{1} x_{2}-14 x_{5}+6 x_{6} \geq 0 \text {, } \\
& -0.5\left(x_{1}-8\right)^{2}-2\left(x_{2}-4\right)^{2}-3 x_{5}^{2}+x_{6}+30 \geq 0, \\
& 3 x_{1}-6 x_{2}-12\left(x_{9}-8\right)^{2}+7 x_{10} \geq 0, \\
& \text { where }-10 \leq x_{i} \leq 10, i=1,2, \ldots, 10
\end{aligned}
$$

\subsection{Setting of parameters in MPEA}

Control parameters are set as follows:

Population Size: 50 .

The number of independent runs is 20 .

The amount of parents in Multi-Parent crossover operators : $M=4$

Computing precision: $10^{-14}$

\subsection{Analysis of Results}

Table 1. The comparison of this algorithm and the algorithms in the [13] and [14] 


\begin{tabular}{|c|c|c|c|c|c|}
\hline problem & Study & Best & Average & Worst & $\begin{array}{l}\text { Known best } \\
\text { result }\end{array}$ \\
\hline \multirow{3}{*}{$G_{1}$} & MPEA & -31025.560 & -31025.560 & -31025.560 & \multirow[t]{3}{*}{-30665.5} \\
\hline & {$[13]$} & -30665.5 & -30665.5 & -29846.7 & \\
\hline & [14] & No data & No data & No data & \\
\hline \multirow{3}{*}{$G_{2}$} & MPEA & -15 & -15 & -15 & \multirow[t]{3}{*}{-15} \\
\hline & [13] & -15 & -15 & -13 & \\
\hline & [14] & -15 & -15 & -15 & \\
\hline \multirow{3}{*}{$G_{3}$} & MPEA & 7049.2480 & 7051.2780 & 7068.6440 & \multirow[t]{3}{*}{7049.330923} \\
\hline & [13] & 7062.2 & 7220.0 & 10230.8 & \\
\hline & [14] & 7378.0 & 8206.2 & 9653.0 & \\
\hline \multirow{3}{*}{$G_{4}$} & MPEA & 680.630 & 680.630 & 680.630 & \multirow[t]{3}{*}{680.6300} \\
\hline & [13] & 680.634 & 680.642 & 680.651 & \\
\hline & [14] & 680.642 & 680.718 & 680.995 & \\
\hline \multirow{3}{*}{$G_{5}$} & MPEA & 0.05395 & 0.1125 & 0.44025 & \multirow[t]{3}{*}{0.053950} \\
\hline & [13] & 0.05395 & 0.24129 & 0.50776 & \\
\hline & [14] & 0.054 & 0.064 & 0.577 & \\
\hline \multirow{3}{*}{$G_{6}$} & MPEA & 24.3062 & 24.3062 & 24.3062 & \multirow[t]{3}{*}{24.3062091} \\
\hline & [13] & 24.372 & 24.409 & 25.075 & \\
\hline & [14] & 25.486 & 26.905 & 42.358 & \\
\hline
\end{tabular}

1) From the Table 1, we can see that the experimental results using the new algorithm are surprisingly good. For the six benchmark problems, the results of MPEA are better than those using the algorithms proposed in the [13][14] in the best results, average results and worst results, which shows the good performance of the new algorithm in generality, effectiveness and stability.For the problems $G_{2}, G_{4}, G_{5}, G_{6}$ the best results gained by MPEA are same with the known best results .

2) For the problems $G_{1}$, the known best result is -30665.5 . While our new best result that is -31025.560 and the optimum point is

$78.00000000000003,33.00000000000001$, $27.07099710517606 \quad, \quad 44.99999999999996$ 44.96924255010539）。 
3) For the problems $G_{3}$, the known best result is -7049.3307 . While our new best result is 7049.2480 and the optimum point is:

( 579.30669022708838 , 1359.97068853270640 ,

$5109.97064176874688 \quad, \quad 182.01770006567686$,

$295.60117432924806 \quad, \quad 217.98229993432710$,

286.41652573643283, 395.60117432924902)。

4) Generally, the setting of parameters in MPEA is very simple, thus the performance of our algorithm is independent on the value of parameters which is crucial to one algorithm's generality .

5) MPEA can also solve function optimization problems without constraint. Some typical test problems are solved by this algorithm. The results show that MPEA can solve unconstrained function optimization problems with high stability and performance .

\section{CONCLUSIONS}

This paper presents a novel multiple populations evolutionary algorithm (MPEA) to handle the constrained function optimization problems. The MPEA have three populations and each population adopts different multiparent crossover operators. Because of the complementarity among three crossover operators, the diversity of all individuals in each generation is enhanced so that the MPEA is more likely to find the global optimum than other single population evolutionary algorithms. At the same time, different populations exchange their best solutions in each generation during the MPEA runs to direct its search, which improves its search performance ulteriorly. The new approach is compared against other evolutionary optimization techniques in several benchmark functions. The results obtained show that the new approach is a general, effective and robust method.

\section{ACKNOWLEDGEMENTS}

This research is supported by the National Natural Science Foundation of China (No. 60473081,60133010, 40275034). 


\section{REFERENCES}

[1] Storn R And Price K.,Minimizing the real functions of the ICEC'96 contest by differntial evolution.IEEE Conf.on Evolutionary Computation.Nagoya.1997,842 844.

[2] Chiou J P and Wang,P S.A hybid method of differential evolution with application to optimal control problems of a bioprocess system. IEEE Conf. On Evolutionary Computation .Anchorage .1998,627 631

[3]Yun-Chien Lin,Feng-Shen Wang,and Kao-shing Hwang.a hybrid method of evolutionary algorithms for mixed-integer nonlinear optimization problems.Proceedings of the 1999 congress on Evolutionary Computation.Washington,D.C.OSA:IEEE Service Center, 1999,3:2159 2169

[4]Michalewicz Z,Nazhiyath G.Genecop III: A Co-evolutionary Algorithm for Numerical Optimization Problems with Nonlinear Constraints.Proc. of 1995 IEEE Int'l. Conf. on Evolutionary Computation(ICEC'95).Perth,Australia,IEEE Press, 1995, 647 651

[5] Renders,J.-M. and H.Bersini(1994). Hybridizing genetic algorithms with hill-climbing methods for global optimization : Two possible ways. In Z. Michalewicz, J.D.Schaffer, H.P.Schwefel, D.B.Fogel,and H.Kitano(Eds.), Proceedings of the First IEEE International Conference on Evolutionary Computation,pp,IEEE Press, 1994.312 -317.

[6] Runarsson TP, Yao X. Stochastic ranking for constrained evolutionary optimization. IEEE Transactions on Evolutionary Computation, 2000,4(3):284 294.

[7]Deb K, Joshi D, Anand A. Real-Coded evolutionary algorithms with parent-centric recombination. Technical Report, KanGAL Report No.2001003, Kanpur: Indian Institute of Technology, 2001.

[8] Efr'en Mezura-Montes \& Carlos A. Coello Coello:Adding a Diversity echanism to a Simple Evolution Strategy to Solve Constrained Optimization Problems. In Proceedings of the 2003 Congress on Evolutionary Computation (CEC'03), IEEE Press, ADFA, UNSW, Canberra, Australia, 8-12 December 2003. pp. 6-13

[9] Tanese R.Distributed Genetic Algorithms.Proc. of the 3rd Int'l. Conf. on Genetic Algorithms. Morgan Kaufmann,Los Altos, 1989

[10] Potts J C,Giddens T P,Yadav S B.The Development and Evaluation of an Improved Genetic Algorithm Based on Migration and Artificial Selection .IEEE Trans.on SMC, 24(1), 1994.73 86

[11] K. Deb and R.B. Agrawal: Simulated Binary Crossover for Continuous Search Space, Complex Systems, 9, 115-148(1995).

[12]Guo T,Kang L S: A new Evolutionary Algorithm for Function Optimization. Wuhan University Journal of Natural Sciences, 1999,4(4) (In Chinese)

[13] K. Deb. An Efficient Constraint Handling Method for Genetic Algorithms. Computer Methods in Applied Mechanics and Engineering, 2000.186(2/4):311 -338

[14] Z.Michalewicz: Genetic Algorithms,Numerical Optimization, and Constraints, In L.Eshelman (Ed.),Proceedings of the $6^{\text {th }}$ International Conference on Genetic Algorithms,Morgan Kaufmann,San Francisco,1995.151 158 